\title{
Semiconductor band switching by charging a small grain with a single electron
}

\author{
J. W. G. Wildöer \\ Research Institute for Materials, University of Nijmegen, Toernooiveld, 6525 ED Nijmegen, The Netherlands \\ and Department of Applied Physics, Delft University of Technology, P.O. Box 5046, 2600 GA Delft, The Netherlands \\ A. J. A. van Roij \\ Research Institute for Materials, University of Nijmegen, Toernooiveld, 6525 ED Nijmegen, The Netherlands \\ C. J. P. M. Harmans \\ Department of Applied Physics, Delft University of Technology, P.O. Box 5046, 2600 GA Delft, The Netherlands \\ H. van Kempen \\ Research Institute for Materials, University of Nijmegen, Toernooiveld, 6525 ED Nijmegen, The Netherlands
}

(Received 24 October 1995)

\begin{abstract}
We observe a very abrupt change in the electron density in scanning tunneling microscope measurements on an InAs(110) surface in the vicinity of a small conductive grain. The observed features are a consequence of charge quantization on the grain, which acts as a single-electron gate electrode. The band bending at the surface changes stepwise when an extra electron is added to the grain, resulting in a sharp closed curve around the grain in topographic images. Features due to the screening of the residual charge of the grain are also discussed.
\end{abstract}

Charges located close to a semiconductor interface will change its local electronic properties significantly. These charges can, for example, be present at charge traps in oxides near the interface and will cause spatial variations in the band edges of the semiconductor. Similar effects will occur in a system consisting of small charged grains on a semiconductor surface. On such a surface a scanning tunneling microscope (STM) gives the opportunity to study spatially resolved effects in the band bending, associated with one individual charged grain. If the charge on such a grain can be controlled, effects in the band bending induced by one single extra electron should be observable in the vicinity of the grain.

Scanning tunneling microscopes can reveal spatial variations in the local density of states with unprecedented lateral resolution. At low temperatures very small deviations are already observable, which has in recent years yielded, for example, images of the distribution of quasiparticles in a vortex core $^{1}$ and of standing waves of surface electrons. ${ }^{2}$ In these experiments Fermi energies are large and the influence of the tip on the local density of states at the surface is negligible. At surfaces, however, where the density of states around the Fermi energy is sensitive for electrical fields, the presence of the STM tip will have a local influence. This is the case with semiconductors, but also with systems where charging energies play a role. The influence complicates the interpretation of spectroscopic data, but, on the other hand, can be used intentionally to vary the electrostatic potential of a surface very locally.

In this paper we present STM measurements showing abrupt changes in the band bending, caused by charging a small grain on an InAs(110) surface. Charges are induced on the grain by the electrostatic action of the tip, which is simultaneously used to probe the changes in the electron den- sity at the semiconductor surface. This double use of the tip is possible since the electric field of the tip extends over a far larger area than the area where the tunneling takes place. We observe a very abrupt change in the conduction electron density on the InAs(110) surface when the tip is in the vicinity of a small conductive grain. The change in the density of states is very clearly visible as a sharp circlelike closed curve in topographic images [Fig. 1(a)], which also show the atomic lattice of the InAs(110) surface. We explain the observed features as a consequence of charge quantization on the small grain.

Charge quantization experiments performed with a STM are usually done in a double junction geometry. ${ }^{3-6}$ The tip then acts as one of the leads to a small metallic grain, which is situated on a thin insulating layer on top of a conducting substrate (this is the other lead). If the capacitance of the grain to its environment is sufficiently small, the charging energy dominates the electron transport. The advantage of using a STM to form these geometries is that the tunnel resistance and the capacitance of one of the junctions can be varied by changing the position of the tip. In practice, however, the capacitance can be varied only by a small amount, since the tunnel current falls off exponentially when the tipgrain distance is increased.

In the experiment described here, charges are detected indirectly and therefore also the tip-to-grain capacitance can be varied substantially. We will show that the circle observed in Fig. 1(a) is a line of constant tip-to-grain capacitance and in this way images the electric field of the STM tip.

The InAs used in this experiment is lightly $n$ doped, $n=2.5 \times 10^{16} \mathrm{~cm}^{-3}$ with a Fermi energy of $10 \mathrm{meV}$. The (110) surface was obtained by in situ cleaving the crystal at low temperature with a small sledge mounted on the sample 

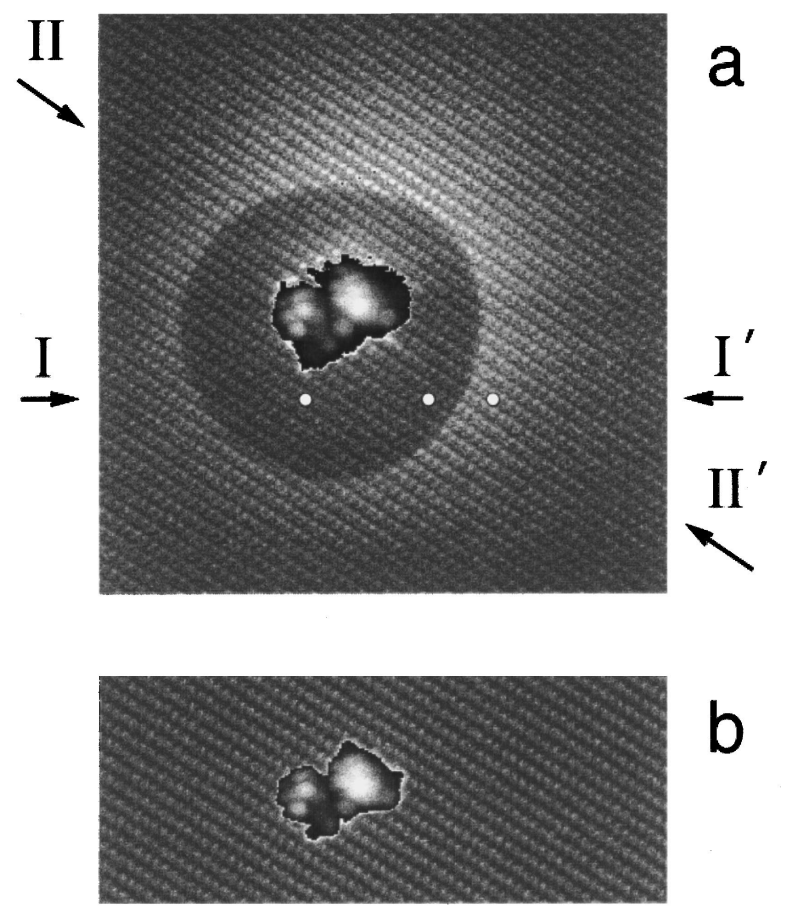

FIG. 1. Constant-current STM images at sample voltages of (a) $-400 \mathrm{mV}$ and (b) $400 \mathrm{mV}$. The scan range is $230 \AA^{2}$. (b) is only partially depicted. The tunnel current is $150 \mathrm{pA}$. The arrows I and I' indicate a line along which spectroscopy curves are taken. The three white dots are positions corresponding to the spectroscopy curves shown in Fig. 2(a). For clarity separate gray scales are used for the grain and the InAs. The scales can be deduced from Fig. 4, in which a cross section along the line II-II' is shown. The details in the structure of the grain show that it is conductive. The data are unfiltered.

holder of the STM. The STM was enclosed by a vacuum can that was filled with helium gas to provide sufficient heat exchange with the helium bath. Due to the cold-trap action of the walls of the insert, surfaces stay clean for several days in our setup. The STM used in this experiment is similar to one described elsewhere, ${ }^{7}$ but modified for cleaving at low temperatures. The STM tips were made by cutting $\operatorname{Pt}(90 \%)$ $\operatorname{Ir}(10 \%)$ wires. The temperature during the measurements was $4.2 \mathrm{~K}$.

If cleaving was successful large atomically flat terraces were observed, separated by monoatomic steps. Similar to images of $\mathrm{GaAs}^{8}{ }^{8}$ either In or As atoms are shown, depending on the bias voltage. The surface buckling is also present on the InAs(110) surface. Therefore at relatively low voltages [as used in Figs. 1(a) and 1(b)] both polarities show the As atoms.

The small grain present in Figs. 1(a) and 1(b) was found on a large atomically flat terrace after the feedback system was oscillating for a short time directly after the tip approach. During oscillation tip-sample distances vary with large amplitudes and at some instances the tip can be very close to the sample surface. This can have caused a loosely connected part of the tip to fall down on the sample, since electrostatic forces increase considerably at small distances. ${ }^{9}$ We make, however, no assumptions on the exact nature of the grain, but conclude from our measurements that it is conductive. After half an hour of measuring the grain disap-
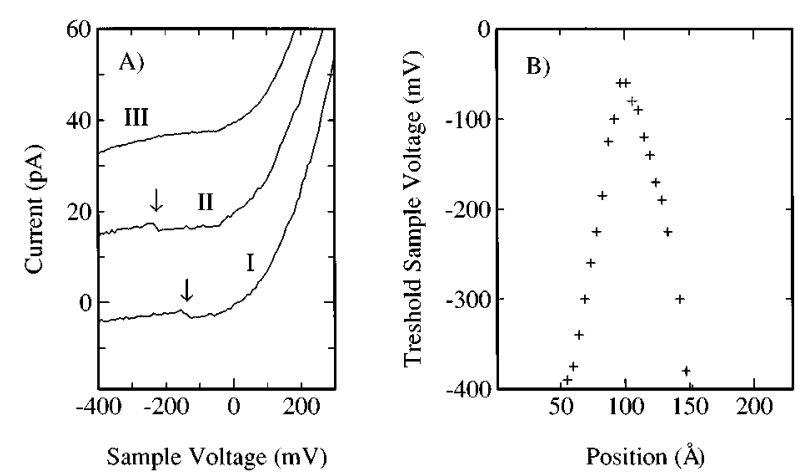

FIG. 2. (a) Spectroscopy curves taken along the line I-I' of Fig. 1(a) at $x=85 \AA$ (I), $x=135 \AA$ (II), and $x=160 \AA$ (III) $[x$ is taken relative to the left-hand side of Fig. 1(a)]. Curves II and III have an offset for clarity. (b) Threshold sample voltage as a function of position along the line I-I' $[x$ is taken relative to the left-hand side of Fig. 1(a)].

peared and was presumably picked up by the tip. After this, the flat surface showed no defects and the other features present in Fig. 1(a) had disappeared. This shows that the observed phenomena are caused solely by the grain and not by defects at the sample surface.

The sharp circle shown by the topographic images can be excluded to originate from a real change in height, since it is only present when the sample voltage is negative. Positive sample voltages only show a regular lattice [Fig. 1(b)]. Also, at the circle in Fig. 1(a), ${ }^{10}$ the underlying lattice is still clearly visible and shows no distortion. A very remarkable feature of the circle is its extreme sharpness. The change in $z$ deflection takes place on a length scale shorter than the lattice constant. Furthermore, it is worth noting that the circle is not located symmetrically around the small grain.

In addition to topographic images, current-voltage $I-V$ curves have been taken along the line I-I' indicated in Fig. 1(a). $I-V$ curves are obtained by interrupting the feedback system and subsequently sweeping the tunnel voltage over a present range in about $0.5 \mathrm{~s}$. The tip height is set with the feedback system active by choosing a tunnel voltage $\left(V_{\text {set }}\right)$ and a reference current $\left(I_{\text {ref }}\right)$. Figure 2(a) shows three $I-V$ curves taken at $x=85 \AA$ (I), $x=135 \AA$ (II), and $x=160 \AA$ (III). In these measurements the tip height was regulated with a sample voltage $V_{\text {set }}=+400 \mathrm{mV}$ (the tip voltage was kept at zero) and a reference current $I_{\text {ref }}=150 \mathrm{pA}$. $V_{\text {set }}$ was chosen at this value to avoid the variations in the local electron density to influence the tip height. With the sample voltage of $+400 \mathrm{mV}$ the Fermi level of the tip is well above the conduction band edge of the InAs, so therefore all $I-V$ curves are taken at the same tip-sample distance.

Since the InAs used is a (degenerate) semiconductor, the shape of the curves is asymmetric. At positive voltages electrons tunnel from the tip into the conduction band and the available number of states for tunneling is much larger than at negative sample voltages. Note that in the gap (negative voltage) the differential conductance is not zero, which indicates tip-induced band bending.

A more remarkable feature is the sharp jump present in curves I and II at negative sample voltages. At the voltage of the jump the differential resistance is negative. Although the 


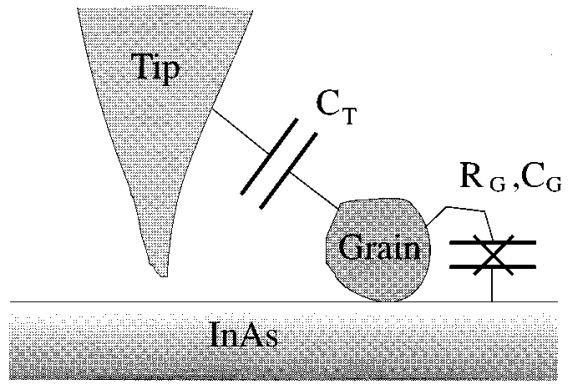

FIG. 3. Representation of the model. The tip induces an extra electron on the grain when the bias voltage exceeds a value determined by the tip-to-grain capacitance $C_{T}$.

effect in the $I-V$ curves is small, it is present in all curves taken within the region of the circle. The bias voltage where the jump takes place varies with position as shown in Fig. 2(b). The voltage increases when the edge of the circle is approached and roughly equals $-400 \mathrm{mV}$ at the edge. Since this is the tunnel voltage that was used in Fig. 1(a), we conclude that the dark area in Fig. 1(a) is defined by the value of the bias voltage and maps the occurrence of a jump in the $I-V$ curves between zero and the bias voltage. From Fig. 2(b) we thus conclude that a smaller bias voltage results in a smaller circle.

The jump in the $I-V$ curves cannot be explained by straightforward tip-induced band bending because of its negative differential resistance and also not by interference effects of the conduction electrons, given the abruptness of the transition in the topographic images (the wavelength of the conduction electrons is of the order of a few nanometers). The shape of the $I-V$ curves indicates a switching of the conduction band between two levels. This switching is induced by the bias voltage between tip and sample and results in a stepwise decrease of the conduction electron density.

We explain the observed features by single-electron charging of the grain induced by the electric field of the tip. The charge on the grain leads to band bending at the semiconductor surface. Because of the quantized nature of the electron charge only discrete levels are possible. The model we use is shown in Fig. 3. The bias voltage required to charge the grain can be given simply by ${ }^{6}$

$$
V_{T}=-\frac{e}{2 C_{T}}\left(1-\frac{2 Q_{0}}{e}\right)+\frac{\Delta W}{e},
$$

where $Q_{0}$ is the offset charge of the grain and $C_{T}$ represents the tip-to-grain capacitance. The work function difference between the tip and the InAs surface is accounted for by the term $\Delta W$. To allow charging, the tunnel resistance from grain to substrate $R_{G}$ has to be larger than $h / e^{2}$. Note that the grain to substrate capacitance $C_{G}$ has no influence in this model. The data of Fig. 2(b) are in agreement with the model, since by increasing the tip-grain distance the capacitance $C_{T}$ is decreased. It is difficult to estimate $Q_{0}$ from these data since the work-function difference $\Delta W$ is unknown, largely because it depends critically on the shape of the tip.

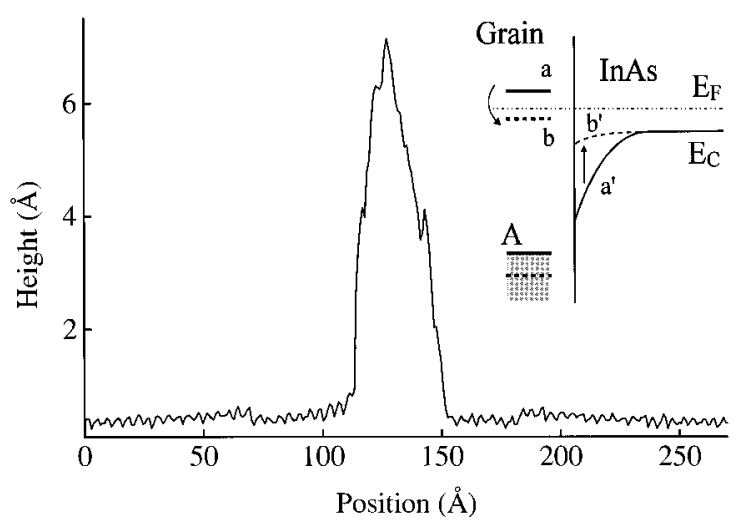

FIG. 4. Cross section along the line II-II' in Fig. 1(a). The depicted cross section is taken through the highest point of the grain, showing its maximum height at approximately $7 \AA$. Further, both the atomic lattice and the height change at the edges of the circle are clearly visible. Inset: band bending in the charged (dashed line) and the uncharged situation. Switching of the conduction band from $a^{\prime}$ to $b^{\prime}$ will occur when the charged state is moved below the Fermi energy. $A$ is the residual charge of the grain.

At the sharp circle of Fig. 1(a) a tip-sample voltage of 400 $\mathrm{mV}$ was required to charge the grain and consequently the circle is a line of constant tip-grain capacitance $C_{T}$. The deviations from the symmetric shape of the circle are a consequence of the shape of the tip. In fact, from this measurement it is possible to extract quantitative information about the shape of the tip. Larger grains will result in smaller charging energies and in that case more circles of equal capacitance should be observable, each one corresponding to a successive integer number of the electrons on the grain.

Apart from the dark area described earlier, also a slowly fading brighter circle is present in Fig. 1(a), which is located symmetrically around the grain. In the brighter circle the conduction electron density is enhanced compared to far away from the grain. Outside the circle the grain is not charged by the tip, but still influences the band bending in the surrounding area via its residual positive charge.

The cross section along the line II-II' indicated in Fig. 1(a) is shown in Fig. 4. On both sides of the grain the sudden increase in tip height is followed by a gradual decrease. In fact the grain closely approximates a screened point charge, for which the potential $\phi(r)$ is given by ${ }^{11}$

$$
\phi(r)=\frac{Q}{r-r_{0}} e^{-\left(r-r_{0}\right) / l_{s}},
$$

where $l_{s}$ is the screening length and $Q$ the point charge at $r_{0}$. The (positive) residual charge results in an increase in the state density below the Fermi energy. In the charged situation, the grain-induced band bending appears to be rather small, since the tip height is almost equal to far away from the grain. The band bending at the surface both in the charged and the uncharged situation are illustrated schematically in the inset of Fig. 4. The band bending is not only determined by the work function difference, as with large interfaces, but also by the charging conditions. The work function difference between grain and InAs determines $Q_{0}$ in (1), whereas the charging energy and the external electrical 
field determine the energies of the charged states in the grain and through these also the actual band bending. Since the fading brighter circle (the uncharged situation) is caused by the residual charge $A$, the influence of the tip on its shape will be of minor importance. Therefore, in contrast to the charging circle, the screening circle has a symmetric shape. The screening length $l_{s}$ can be calculated to be $4.3 \mathrm{~nm} .{ }^{11}$

To image features such as the ones described here properly, both tip and grain shape should be such that no double tip effects occur. Also, changes in the electron density are only present within a small area around the grain, determined by the screening length. Although we observed abrupt transitions also around other grains, the effects were less clear than in the measurements presented here. Attempts to deposit metallic grains in a more controlled way by field evaporating from a gold tip at low temperature were unsuccessful. However, various other techniques for depositing small metallic particles at low temperatures exist and in a more dedicated setup system such as the one described here should be relatively easy to obtain.
In conclusion, we have been able to observe effects in the electron density in a semiconductor associated with the quantized charge of a small grain on its surface. In the vicinity of the grain the conduction band switches between two levels, depending on the charge of the grain. In the experiment the STM is both used to charge the grain and to probe the electron density. In the topographic measurements the tip-to-grain capacitance and the screening area are imaged. The system presented offers the possibility to study charging effects both in a direct and an indirect way, simply by varying the position of the tip. More experimental data can yield quantitative information about $Q_{0}$ in these systems and the role of the work function in the charging process.

This work was supported by the Dutch Foundation for Fundamental Research of Matter FOM, which in turn is financially supported by the Dutch Organization for Scientific Research NWO. We are grateful to C. Schönenberger for the InAs material.
${ }^{1}$ H. F. Hess, R. B. Robinson, and J. V. Waszcak, Phys. Rev. Lett. 64, 2711 (1990).

${ }^{2}$ M. F. Crommie, C. P. Lutz, and D. M. Eigler, Nature 363, 524 (1993).

${ }^{3}$ P. J. M. van Bentum, R. T. M. Smokers, and H. van Kempen, Phys. Rev. Lett. 60, 2543 (1988).

${ }^{4}$ R. Wilkins, E. Ben-Jacob, and R. C. Jaklevic, Phys. Rev. Lett. 63, 801 (1989)

${ }^{5}$ J. G. A. Dubois, E. N. G. Verheijen, J. W. Gerritsen, and H. van Kempen, Phys. Rev. B 48, 11260 (1993).

${ }^{6}$ C. T. Black, M. T. Tuominen, and M. Tinkham, Phys. Rev. B 50,
7888 (1994).

${ }^{7}$ J. W. G. Wildöer, A. J. A. van Roij, H. van Kempen, and C. J. P. M. Harmans, Rev. Sci. Instrum. 65, 2849 (1994).

${ }^{8}$ R. M. Feenstra, J. A. Stroscio, J. Tersoff, and A. P. Fein, Phys. Rev. Lett. 58, 1192 (1987).

${ }^{9}$ Oscillating the feedback system is often done deliberately to obtain stable tips.

${ }^{10}$ The shape and size of the circle are reproduced exactly in images taken over half an hour.

${ }^{11}$ See, for example, C. Kittel, Introduction to Solid State Physics (Wiley, New York, 1971). 